\title{
Implicit learning in rule induction and problem solving
}

\author{
Aldo Zanga, Jean-François Richard, and Charles Tijus \\ Université Paris 8, France
}

\begin{abstract}
Using the Chinese Ring Puzzle (Kotovsky \& Simon, 1990; P. J. Reber \& Kotovsky, 1997), we studied the effect on rule discovery of having to plan actions or not in order to reach a goal state. This was done by asking participants to predict legal moves as in implicit learning tasks (Experiment 1) and by asking participants to make legal moves as in problem-solving tasks (Experiment 2). Our hypothesis was that having a specific goal state to reach has a dual effect on rule discovery. The first effect is positive and related to feedback from moves done in order to attain the goal: generalising the results of action and associating them to the conditions in which they were obtained allows discovery of the rule and learning it. The second effect is negative. In attempting to reach a specific goal, participants first tend to reduce the distance that separates the current state from the goal state (hill climbing) and so neglect the kind of exploration that facilitates rule and procedure discovery because this would seem to be a detour from the goal. Results show that having to plan actions improved performance in implicit learning tasks (Experiment 1), yet it impaired performance in problem-solving tasks (Experiment 2). Although implicit learning and problem solving are based on rule discovery, and entail noticing regularities in the material, in both cases, rule discovery processes appear to be task-dependent.
\end{abstract}

Solving a problem entails transforming material in an initial state into a specific goal state. For instance, in the familiar Missionaries and Cannibals problem $(\mathrm{M} \& \mathrm{C})$ three missionaries and three cannibals initially on the left bank of a river must be transported to the right bank. Whether transformations are discontinuous as in the Tower of Hanoi problem $(\mathrm{TOH})$ or continuous as in the Nine Dots problem (NDP) there are in both cases constraints on transformation that can be stated in the form of rules (Figure 1): "Only one disk can be moved at a time", "Only the top disk on a peg can be moved", and "a larger disk cannot be placed on a smaller disk"

Correspondence should be addressed to Aldo Zanga, Laboratoire CNRS-FRE 2627 "Cognition \& Usages" Université de Paris 8, 2, rue de la Liberté, 93526 Saint-Denis Cedex 02, France. Email: tijus@univ-paris8.fr

The research reported herein was supported by the CNRS.

(C) 2004 Psychology Press Ltd

http://www.tandf.co.uk/journals/pp/13546783.html

DOI: $10.1080 / 13546780342000034$ 
(TOH); "You cannot draw over an existing line", "The lines must be continuous (you cannot lift the pencil from the paper)", "Some lines should extend beyond the area that contains the dots" (NDP).

Once the initial state, the goal or final state and the rules for transforming the variable properties are known, one can derive the Problem Space (or "Search Space") which is the finite set of all the various states through which the problem solver can move on the different paths to solution (Newell \& Simon, 1972) and represent it in the form of a graph where each move produces another state, called a "node". For instance, the TOH graph is made up of 27 states, or nodes, and the Chinese Ring Puzzle (ChiRP) that we have chosen for our study (Figure 2), contains 31 (Kotovsky \& Simon, 1990).

Historically, a distinction has been made between ill-defined and welldefined problems. A well-defined problem is a "construction" problem in

Nine dots problem Tower of Hanoi Problem

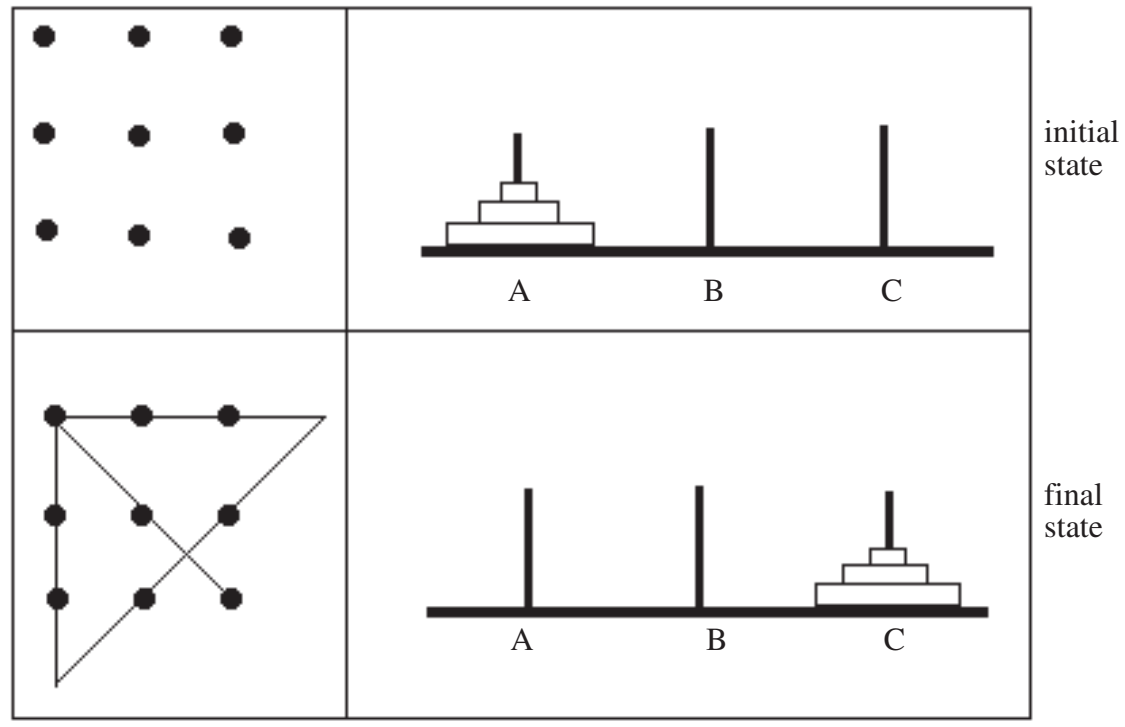

Figure 1. Initial state and goal state for the Nine Dots problem and the Tower of Hanoi problem (TOH). In the Nine Dots problem, the nine dots are not joined in the initial state. In the goal state, four straight lines should join them. The dots have permanent properties (colour, size, and location) and variable properties (being or not being linked). In TOH, all the disks are on Peg A in the initial state, and in the goal state they are all on Peg C. The disks have permanent properties (colour and size) that cannot be changed and variable properties that need to be transformed (peg location, position in the stack). In order to solve both these problems, the variable properties need to be transformed. 


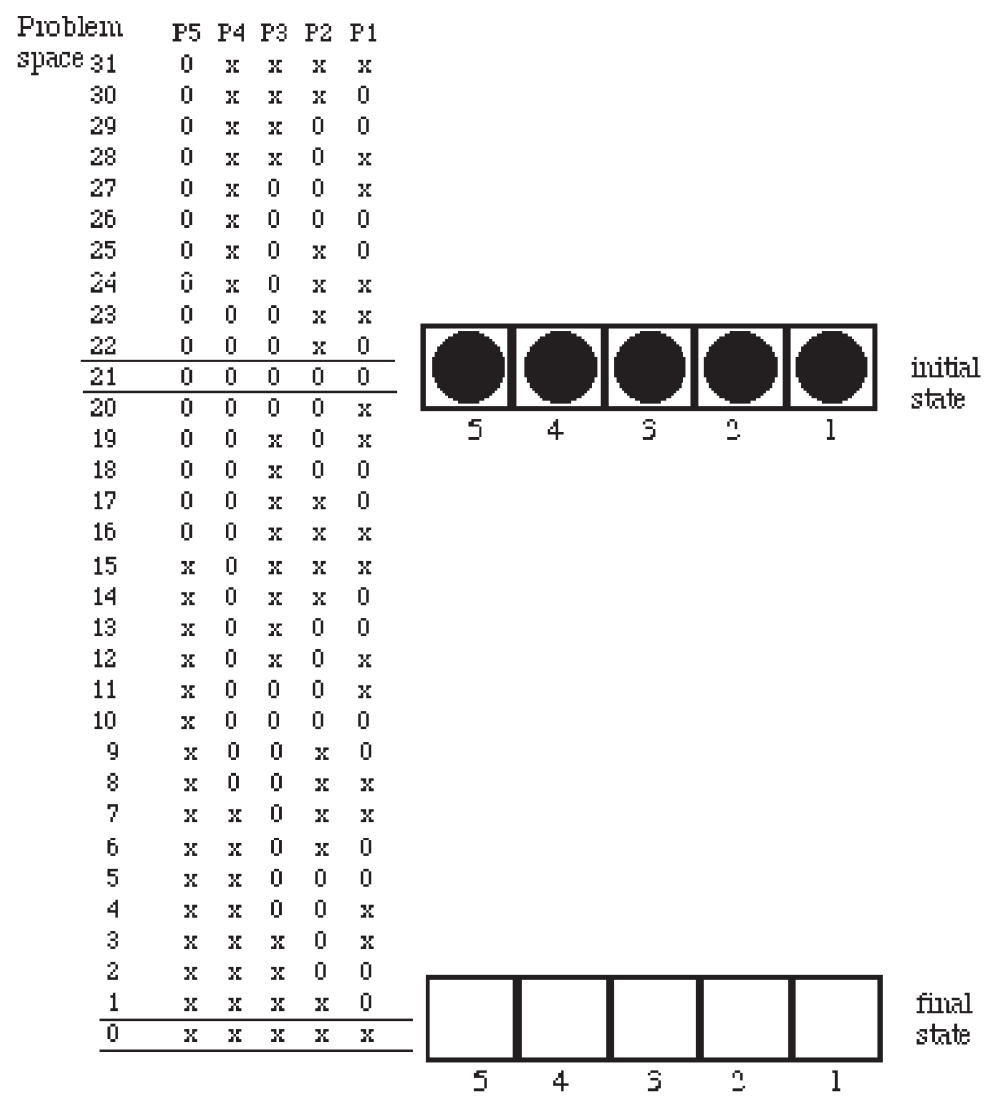

Figure 2. The graph of states, or problem space, for the Chinese Ring Puzzle (ChiRP): "O" in the graph indicates the presence of a token in a slot; " $x$ " indicates the absence of a token. On the left is the representation of all the possible states ( 31 to 0 ). On the upper right is the initial state as the participant sees it on the computer screen, and on the lower right, the goal state. The material is composed of five slots, each holding a token $(\mathrm{O})$ in the initial state (21). To reach the goal state, all the tokens must be removed. The condition for removing or placing a token in a slot is that there be a single token to the right of that slot and none beyond. Theoretically, it is a simple problem to solve because there is only one path to the goal state.

which the participant has access to all the information he or she needs, including a specific goal, which is described in the form of a state and the rules for transformation. This is the case for TOH (Figure 1). An ill-defined problem is a "design" problem in which a non-specific goal is given in the form of constraints and some necessary information is withheld from the participant. This is the case for the Nine Dots problem, as shown in Figure 1: the participants possess an implicit constraint to stay within the dotted area. 
This distinction made between two kinds of problems has led to consideration that there are two kinds of problem-solving processes: planning and memorising under the constraint of cognitive load for welldefined problems (Anzaï \& Simon, 1979; Kotovsky \& Kushmerick, 1991; Simon, 1979), creativity, insight, and restructuring mental representations of the situation (and particularly the objects it comprises) for ill-defined problems (Duncker, 1945; Kohler, 1964; Lockhart, Lamon, \& Gick, 1988; Mayer, 1983; Ohlsson, 1984a, 1984b).

Richard, Poitrenaud, and Tijus (1993) have called this distinction into question. On the one hand, protocols for ill-defined problems such as, for instance, the Nine Dots problem (NDP), show that participants solve the problem in two phases, after 30 to 50 trials - which is to say, after having eliminated one by one all of the possibilities in which the lines do not extend beyond the dots (MacGregor, Ormerod, \& Chronicle, 2001; Tijus \& Najab, 1986; Weisberg \& Alba, 1981). It is only after this first phase that participants restructure their representations, consider extending the lines beyond the area mapped out by the dots, and then move quickly, often suddenly, on to solution. Two phases have also been attested for welldefined and very difficult problems: after an often lengthy exploratory phase involving many errors, participants move on to a second and brief phase that is almost errorless (Figure 3). Much exploration is often involved in solving certain $\mathrm{TOH}$ isomorphs - tasks packaged differently from the classic and well-defined $\mathrm{TOH}$ problem but whose problem spaces are identical. Some of these require up to 120 moves when 7 are enough (Kotovsky, Hayes, \& Simon, 1985). Similarly, solving ChiRP sometimes requires up to 500 moves when 21 are enough (Kotovsky \& Simon, 1990).

In solving well-defined problems, the phase of trial and error was at first perceived as a planning phase: in attempting to reach the goal, the participant experiments because he or she needs to mentally construct the solution path by constructing and memorising a stack of subgoals. However this hypothesis neither explains why participants break the rules they were given nor why some isomorphs are so much more difficult to solve, despite the fact that planning their solutions is considered equally difficult. Simon (1979) explains the difference in difficulty between isomorphs of the "Monster" problem and more specifically, between "change" problems and "transfer" problems, by a difference in cognitive load. We challenge this explanation and will come back to it.

The exploratory phase is now thought to be a phase of learning to "understand" the rules for moves: learning "what constitutes a move" (Clément \& Richard, 1997; Kotovsky \& Simon, 1990; P. J. Reber \& Kotovsky; 1997; Richard, Clément, \& Tijus, 2002; Richard et al., 1993). However, difficulty in learning "what makes a move" can be related either to memory load or to the induction of the underlying rules. 


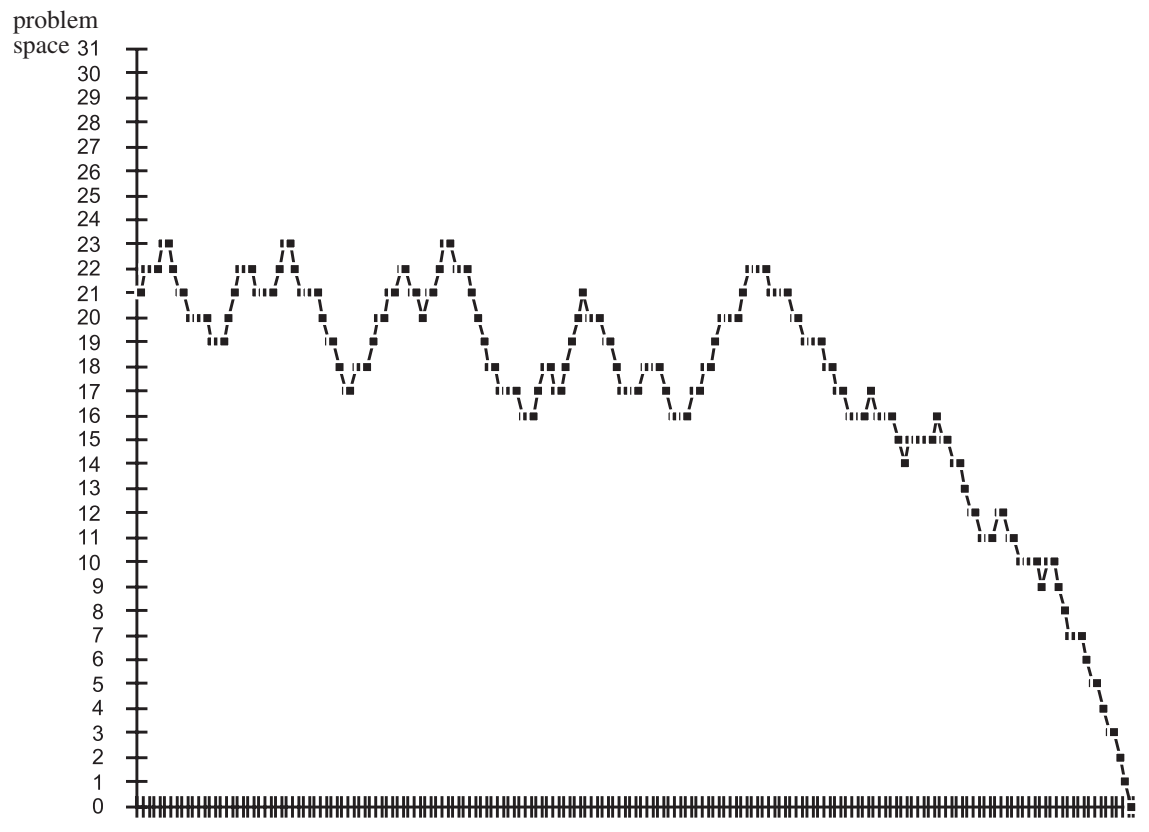

moves

Figure 3. A typical example of ChiRP solution by a participant: for each trial, the figure shows the position on the graph shown in Figure 2 (from state 31 to state 0 ). After an exploratory phase of trial and error, 150 trials in this case, the participant moves into a second phase that shows few errors and little backtracking. This protocol, collected from one of our participants, resembles protocols furnished by Kotovsky and Simon (1990).

In their studies of the Chinese Ring problem and transfer between isomorphs, Kotovsky and Simon (1990) found that the principal difficulty the participants encounter is one of learning "what makes a move", in other words, the criteria that make it possible to effect a change in the material. Nonetheless, "what makes a move" is exactly the kind of information one finds in the verbal instructions to a problem. The authors found that giving visual cues about a move's legality facilitates both transfer and the problemsolving process. Although they do not indicate precisely what facilitates or inhibits discovering "what makes a move", they do make a number of crucial observations about the process of discovering "what makes a move".

Kotovsky and Simon report, for instance, that solving the problem is made easier when participants begin with state 31 (Oxxxx) despite the fact that this lengthens the problem space (1990, p.170). Participants who begin with state 31 are forced to begin by replacing tokens and necessarily learn that in order to remove a given token others must be added. Although 
distance to goal is $50 \%$ longer from state 31 than from state 21 as measured on the problem space, participants starting at state 31 do not require $50 \%$ more moves in order to solve the problem. The authors found no significant difference in the number of moves required to solve the problem between participants starting at state 31 and participants starting at state 21, although success rates were slightly higher for participants starting at state 31.

Another one of the authors' important observations is that state 15 (xOxxx) represents a turning point in the solving process: participants often hesitate here and then make a final sprint towards the goal. However, what the authors did not note is that the way the tokens are arranged in state 16 (OOxxx) provides the ideal situation in which to learn that "You can withdraw (or add) a token" (t5), "if there is a single token to its right" (t4), "and none beyond". Indeed, discovering "what makes a move" is discovering a rule that can be applied to tokens in a wide variety of patterns and this can only be done through abstraction and inference through inductive processes. In certain states, certain tokens illustrate the rules better than others because they have more of the properties stated in the instructions: token $\mathrm{t} 5$ in state 16 has exactly the properties stated in the rules; in state 1 (OOOOO) token $\mathrm{t} 1$ has none of them and token $\mathrm{t} 2$ has one of them (a token, t1, to its right) but not the others (there is no empty slot further to the right) and it has supplementary properties about which the instructions say nothing (it has three tokens to its left).

Further, Kotovsky and Simon also report that participants appear to choose states which lead to other states that superficially resemble the goal but that in terms of problem space are farther from the goal, something Jeffries, Polson, and Razran (1977) also found-which is to say that participants tend to remove tokens in order to arrive at a state that looks like the goal or that is "perceptually" close to it.

The fact that starting at state 31 improved performance, and that state 15 represented a turning point, are facts that support an understanding approach of "what makes a move" by the discovery of the underlying rules, while perceptively close-to-the-goal based behaviour might impair induction.

At the beginning of a difficult problem, the rules given in the instructions are not always fully understood: participants do not see how the general rules given in the instructions apply to the material at hand. Consider, for instance, the ChiRP rule given in Figure 2: "You can withdraw or add a token if there is a single token to its right and none beyond". First of all, why would anyone want to place a token in a slot when the slots are all filled, state 21 (OOOOO), and the aim is to remove them? Second, what does "a single token to its right" mean? It should be understood as "when there is a token in the right-hand slot" but participants often interpret it as meaning 
"when there is a token in any of the slots to the right". Furthermore, what does "and none beyond" mean? This should be understood as "all of the slots, if there are any, must be empty if they are located to the right of the token that is immediately to the right of the token to be removed." Participants often interpret "and none beyond" as meaning that "there must be slots to the right and that these must be empty". This interpretation of the instructions makes it impossible to withdraw token 1, because there are no tokens to its right. The other four fulfil the first condition as understood, they all have a token to the right, but not the second condition, because none of them has an empty slot beyond. This is why, after reading the instructions, for some participants, "It is impossible to make a move!"

This lack of understanding can be found in the exploratory phase: moves that break the explicit rules show that participants do not understand that the rules are applicable to those moves. This lack of understanding is also revealed by the fact that participants self-impose further restrictions (Richard, 1982; Richard et al., 1993): there are moves a participant will not consider because he or she thinks they are not allowed. Participants give themselves supplementary constraints by considering certain moves to be forbidden when they are in fact legal. These implicit constraints, as opposed to the explicit constraints that are given in the instructions, make the problem more difficult. This happens, for instance, with $\mathrm{TOH}$, when a participant thinks that to move a disk from $\mathrm{A}$ to $\mathrm{C}$, it must first be placed on B. Implicit constraints can also generate impasses, for example, in $\mathrm{TOH}$, when the participants thinks that a disk cannot be moved from $\mathrm{A}$ to $\mathrm{C}$ when $\mathrm{B}$ is blocked, or for instance in ChiRP, when participants conclude that no token can be withdrawn at state 21 (OOOOO).

We claim that during the exploratory phase, participants discover the rules and suddenly apply them during the last phase. A typical problemsolving process takes place as if participants were discovering the rules during a trial and error phase and then suddenly putting what they had learned to work in the final phase, as in Figure 3 (Kotovsky \& Simon, 1990). From our point of view, the "errors" found in the exploratory phase are actions that participants think are or might be legal. The participant either believes the erroneous move to be legal or is testing its legality.

To discover the rules of a problem, participants often benefit from situations of impasse generated by the implicit constraints they have given themselves. When they no longer know what to do, participants will either abandon the problem or try making moves they believe to be illegal and so discover new properties that make action possible. The new properties are then used to describe the objects (Richard et al., 1993). For example, a participant who thinks no action is possible at state 21 , the initial state of ChiRP (OOOOO), can try successively removing each and every token beginning on the far left with $\mathrm{t} 5$, then $\mathrm{t} 4, \mathrm{t} 3$, $\mathrm{t} 2$, and $\mathrm{t} 1$. He will end up with 
(OOOxx) and so believe that a token can be withdrawn when it is the first or the second from the right. Taking these new properties as the conditions for withdrawing a token, he will try removing $\mathrm{t} 3$ and $\mathrm{t} 4$, the first two tokens of the new pattern (OOOxx), an action that will result in OxOxx, and will not be able to understand why t3 cannot be removed, which is to say: "what makes a move".

Our point of view is that the rule that works for all of the states, the one Kotovsky and Simon provide, cannot as stated be applied directly to the material in order to define what makes a move legal or illegal. Although additional cues, such as underlining the slots where a change can be made, OOxx $\underline{x}$ for state 16 and OOOOO for state 21, do show where a move can be made successfully and keep the participant from attempting to make moves in other slots, they do not indicate what makes a move legal. Despite such cues, the rule remains inefficient because participants do not understand how to apply it to the material. We are not suggesting that the problem here is one of formulating clear instructions, what we are suggesting is that rules in general often need to be discovered while acting before they can be fully understood. For example in the Chinese Ring problem, the rule, stated or unstated, needs to be discovered. We believe that studies of implicit learning have everything to gain from focusing on the process of rule discovery in situations where participants must manipulate the material themselves in order to reach a goal.

The rule to be discovered can be more or less complex. One can imagine that if ChiRP's rule were "you can withdraw the farthest token on the right or add a token to the slot immediately following it", the problem would be easy to solve. The rules of puzzle-type problems (ChiRP, TOH, M\&C) are complex and make for recursive solving processes that require distancing oneself from the goal in order to reach it. In $\mathrm{TOH}$ for instance, the smallest disk must be placed on peg $\mathrm{C}$ in order to free the largest disk so that it can finally be placed on peg C. In ChiRP, tokens have to be added in order to remove them. In the Missionaries and Cannibals problem, all the cannibals must first be moved to the target bank where the missionaries, whose lives are at risk, are supposed to go.

Keeping peg $\mathrm{C}$ free for the largest disk (TOH), not wanting to replace a token one has already removed (ChiRP), trying from the outset to get all the missionaries safely away from the cannibals and on to the target bank $(\mathrm{M} \& \mathrm{C})$ are all obstacles to solving these problems even though they would appear to help in solving them. The paradox is that it is only in the process of solving/after having solved the problem - which is to say, in the process of constructing the goal/after having constructed the goal-that one discovers how to go about solving the problem, what the rule is, and what makes a move legal. 
Nevertheless, the exploratory phase that allows discovery of the rules has not been extensively studied. As P. J. Reber and Kotovsky (1997) notice, it appears to be very close to implicit learning situations. With a less difficult display than the original version, P. J. Reber and Kotovsky (1997) show that WM (working memory) load slows learning performance. In their first experiment, four groups of participants had to solve the ChiRP twice. When solving the problem the first time, the first group had also to respond when hearing a particular sound with the last of the letters in sequence. The second group was given the same additional task, but with two letters. The third group had to recall three letters. Finally the fourth group, as a control group, solved the ChiRP with no additional task. After solving the puzzle the first time, participants were asked to write about how they solved it, how it worked, and anything to help someone else solve it. Results showed that the more memory is loaded, the greater is the number of moves required to solve the problem. The written retrospective protocols indicated that participants were not able to specify how they had solved the puzzle. Each verbal protocol was rated as to how close to a complete solution strategy the participant came. This rating was on a scale from 1 (no useful information about the puzzle) to 5 (a complete strategy for solving the puzzle). No protocol earned a 5 rating and the majority $(76 \%)$ were rated as 2 or less. Expression of knowledge of the structure of the problem space was almost completely absent, and statements related to explicit strategies were rare at best partial or vague. So, “... the majority of participants are relying exclusively on implicitly acquired information" (P. J. Reber \& Kotovsky, 1997, p. 185). This means that solving ChiRP, although it is a problemsolving task, appears to be based on implicit learning. For the second trial, each group was split and half the participants solved the problem in a zero WM load control condition, whereas the other half solved the problem under a WM load of two letters. Results showed that WM load had no effect on this second trial.

Discovering the rules through induction might correspond to the implicit learning P. J. Reber and Kotovsky (1997) revealed. Two major kinds of implicit learning situations have been largely studied, those that are based on artificial grammars and those that are based on the interaction of variables. In experimental situations of implicit learning based on artificial grammar (A. S. Reber, 1989; A.S. Reber, Kassin, Lewis, \& Cantor, 1980) participants are asked to decide whether or not a string of letters corresponds to another string presented earlier. Some of the strings are constructed randomly and some of them are based on an artificial grammar. In other studies, the task consists of predicting when a target, generated by an automaton, will appear (Lewicki, Hill, \& Czyzewska, 1992; Perruchet, Galleco, \& Pacteau, 1992). The task may for example consist of predicting when the target will appear in one of four quadrants. In one experimental 
condition the target is generated according to a pre-established order (12 sequences out of 240 trials) and in another it appears at random (5 sequences out of 240).

In experimental situations of implicit learning based on interaction between variables, participants are required to reach and maintain the value of an output variable by varying a single input variable, whose effect is contingent on a third variable which is not communicated to them (Berry \& Broadbent, 1984, 1988). The aim of these computer-implemented tasks is, for example, to make a "computer person" describe and continue to describe himself as being "very friendly". The qualifier is chosen from among a list of 12: "very rude", "rude", "very cool", "cool", "indifferent", "polite", "very polite", "friendly", "very friendly", "affectionate", "very affectionate", and "loving". The computer person always begins with the output qualifier "polite" and the participant then replies with an input qualifier of his or her choice. The computer person's response is selected using a variant of the equation $\mathrm{P}=\mathrm{S}-2$ (where " $\mathrm{S}$ " is the number of the qualifier selected by the participant in the order of qualifiers) to which a random element is introduced by having the computer add 1,0 , or -1 to $\mathrm{P}$. This response procedure $(\mathrm{P}=\mathrm{S}-2)$ is not communicated to the participant. His or her task consists of implicitly learning this underlying mathematical rule in order to get the computer to output the right qualifier.

In both artificial grammar and variable interaction types of situations, the findings the authors report show that implicit learning does indeed take place. But how do participants extract rules from the experimental materials that are presented to them? For Perruchet and Amorim (1992), who studied situations similar to those studied by Lewicki et al. (1992), participants process the underlying repetitive structures. For A. S. Reber et al. (1980), implicit learning is a kind of abstraction process that allows participants to integrate the rules that structure the sequences of letters. For Zanga and Perruchet (Zanga, 1997), where patterns of geometric figures are concerned, rule extraction is a kind of implicit memorisation: participants proceed by analogy from the learned sequences to the new ones that are presented to them.

None of these authors found that knowing that there is a rule to discover improves performance. For example, Perruchet et al. (1992) found no significant difference between performances obtained for their "incidental" (implicit condition) group and their "intentional" (explicit condition) group, and some authors have even found that performance is weakened when discovering a rule is made into an explicit task (Berry \& Broadbent, 1984, 1988; A. S. Reber et al., 1980). Finally, dissociation between performance and verbalisations is generally observed, both for tests of similarity judgement (A. S. Reber, 1989) and for tests of variable management (Berry \& Broadbent, 1984, 1988; Mathews, Buss, Chinn, \& Stanley, 1988). 
One of our hypotheses is that participants who attempt to solve a problem without having understood the rules expressed in the instructions behave like participants who are trying to discover a rule: they extract the characteristics of a state from the results of actions and use these as rules. If this is the case, reaching a goal state should play an important role in discovering the rules of a problem, because how close the current state is to the goal state is one of the properties participants might use to evaluate moves.

For instance, Sweller, Mawer, and Ward (1983) have found that novices with insufficient knowledge to construct an appropriate physical representation, cannot work forward from the givens and use means-ends analysis (choosing an equation containing the goal) to guide them to solution. A total of 14 participants were given 25 kinematics problems (for example: "In $18 \mathrm{sec}$ a racing car can start from rest and travel $305.1 \mathrm{~m}$. How fast will it be going when it reaches $305.1 \mathrm{~m}$ ?'). Only two participants worked forward and the others, who tended to work backward on the initial problems, demonstrated an increased tendency to work forward on the last five problems (Experiment 1). With their second experiment, the authors showed an effect of knowing or not knowing the final specific goal of the problem ("What speed will it reach" for the goal group and "Calculate the value of as many variables as you can" for the no-goal group). The specific goal group increased the mean number of problems on which they worked forward compared to the nonspecific goal group.

Tijus and Santolini (1996) found that when participants are asked to draw the Berlin Wall that once separated East and West Berlin, $82 \%$ of participants draw a single straight line for a wall instead of a wall enclosing West Berlin, despite the fact that they know the function of the wall was to prevent people from the East from entering West Berlin. However, when participants are asked to draw a wall that will keep East Germans from entering West Berlin, 52\% draw a wall around the western part of the city. What the authors observed was that the participants who are given the goal will begin by drawing a straight line for the wall and will then modify their drawings when they realise that the drawings do not satisfy the goal.

However, if we conceive the exploratory phase as a mixture of understanding and planning, a precise goal may have a dual effect: it might favour rule discovery (understanding) because the rules must be respected in order to reach the goal, and it might impair performance (planning) when moves are done to reduce the distance that separates the current state from the goal state. This paper explores this dual effect of a specific goal.

Although a central question is how and what people learn while they try to reach a goal, induction and planning, two components of problem solving, rarely interrelate in the literature. As seen above, the distinction between ill-defined (induction) and well-defined (planning) problems was 
of no help, although people can learn more than procedures from problem solving. For Simon and Lea (1974) for instance, a task can be either an induction task or a planning task. In contrast, Vollmeyer, Burns, and Holyoak (1996) espouse a mixture of induction and planning. Using an experimental situation based on interaction between variables (a biologylab system), they demonstrated that providing participants with a specific goal (having a precise number of crabs, prawns, lobster, and sea bass) impaired discovering how the variables interact, while having a nonspecific goal (free exploration) induced a greater overall knowledge of the system structure. Thus, in a quite implicit-like learning task, they found evidence that a specific goal has a negative effect on induction, a result found by Geddes and Stevenson (1997) with a Broadbent dynamic control task, and by Sweller (1988)in a variety of different problems. For instance, Sweller showed that participants given the specific goal of calculating a particular angle, with the subgoals of calculating all angles, were less successful in subsequent problems than participants who were asked to calculate all possible angles. For Sweller, induction and planning are two distinct processes and having to monitor goals increases the cognitive load of the task.

However, do we need a dual-space model? There are at least two reasons why planning moves might impair induction. First, feedbacks are more negative than positive when planning with illegal rules in a situation in which people expect positive results. Second, allocation of attention is greater for the goal at hand than for subgoals, although errors would be on subgoals. Because people act to gain an expected result based on their understanding of "how it works", when planning they pay more attention to their current goal than to the subgoals they are performing. Having to calculate a precise angle, as in the Sweller task, they pay less attention to each of the angles they are calculating. While solving ChiRP, suppose a participant thinks that the five tokens have to be withdrawn from 1 to 5 , withdrawing token 5 being the last move. S/he starts playing token 1, token 2 , token 3 , and token 4 with little attention to intermediate states. More attention might be allocated if the task was a nonspecific goal such as withdrawing each of five tokens in turn. A way of obliging participants to pay attention to subgoals could be to delay actions by implementing an internal cognitive cost on each subgoal, as was done by O'Hara and Payne (1998).

In contrast, imagine a task of observing and predicting the moves carried out by an expert with the full knowledge of the precise goal state. More attention should be paid to intermediate states as positive examples of prerequisites that satisfy the goal.

The object of this article is twofold. First, it is to show that goals (meaning having a goal state to reach) can have a positive effect on rule 
discovery because the rules must be respected in order to construct the goal. Repeated and progressive attempts to construct the goal thus lead to discovering the rules. In $\mathrm{TOH}$, for example, in order to move each of the three disks to peg $\mathrm{C}$, the largest disk must first be placed on $\mathrm{C}$, because a larger disk cannot be placed on top of a smaller one. Thus, constructing the goal is participant to discovering the rule. In ChiRP, in order to remove a token there must be a token to the right of the one to be removed: therefore the first subgoal to construct is removing token 5 (the token furthest to the left) because it is no help in removing any other token.

Second, we wish to show that the goal also has a negative effect. The goal can skew a novice's attention. This is particularly true if he or she mistakes states that only look like the goal state for states close to the goal state. This can lead to inadequate subgoaling that is no help in discovering the rules.

We studied the positive effects of goals (Experiment 1) in a situation provided by ChiRP that is close to implicit learning situations. Participants watch the computer as it runs through a series of legal moves, placing and withdrawing tokens. The participant's task is to predict the computer's next move. In one experimental condition, the computer plays to reach the goal. In the second experimental condition, the computer respects the rules and runs back and forth through the problem space (Figure 2) without necessarily reaching the goal of removing all of the tokens: it moves alternately towards state 0 or towards state 31 and changes direction at random. If goals have a positive effect on rule discovery, then participants in the first condition should have more success in predicting the computer's next move.

Varying goal-specificity (Sweller, 1988), the negative effects of goals were studied by asking participants to make moves, placing and withdrawing tokens (Experiment 2). In one condition, the participants are asked to reach the goal of removing all of the tokens from the playing board. In a second condition, participants are not asked to reach a goal state but rather to play with the apparatus until they have moved each of the five tokens at least once. If goals have a negative effect on rule discovery, participants in the second group should have greater success in learning the rules that allow movement of the tokens.

\section{EXPERIMENT 1: EFFECT OF THE GOAL ON INDUCING LEGAL MOVES}

The object of this experiment is to study the effect of having a specific goal state to reach, on rule discovery in situations where the participant is not asked to solve the problem but rather to predict the computer's next move.

The computer runs through the problem space (Figure 2), placing and removing tokens according to the rules. Progressive rule discovery 
on the participant's part is measured by the accuracy of his or her predictions. We consider that the better the participant's predictions, the more he or she understands the rules, because there is only one legal move possible for each problem state if the move that undoes the previous move (backward through the problem space) is excluded. In the first experimental condition, the computer plays in order to reach the specific goal of removing all of the tokens. This is the condition with a specific goal (SG). In the second experimental condition, the computer runs back and forth through the problem space at random without attempting to reach the goal; this is the nonspecific goal condition (NG).

In both conditions, after having predicted the computer move, participants notice the move actually made by the computer. This procedure can be seen as an implicit learning paradigm of a complex rule underlying visual stimuli presentation.

If goals have the effect of structuring the actions and properties that make moves possible, then participants in the SG condition should perform better than participants in the NG condition.

\section{Method}

Participants. Participants were recruited from the student body of the University Paris 8 and assigned in alternating order to one of the two experimental groups: 10 participants were placed in the SG situation and 10 others in the NG situation. None of them had ever seen the problem before.

Materials. Two automatic versions of the Chinese Ring Puzzle were developed for this experiment. In both versions, the computer removes and places tokens according to the rules (Figure 2). In the first version, used for the SG condition, the computer selects a path that leads to state 0 (Figure 2), the goal state, in which all of the tokens have been removed. When this goal has been reached, the computer selects at random one of the 31 states and then proceeds again towards the goal. In the second version, the one used in the NG condition, the computer runs up and down the problem space graph (Figure 2) either towards or away from the goal. When it reaches the goal (state 0), or the impasse (state 31), or a randomly chosen state reached after having completed at least 21 moves in the same direction, the computer reverses direction.

In both conditions, the participant's task is to predict the computer's move with the mouse by clicking on the slot where he or she believes 
the computer is about to play. Clicking on a slot that contains a token means that the participant believes the computer is about to remove that token. Clicking on an empty slot means that the participant believes the computer is about to place a token there. For each trial, the slot on which the participant has clicked shows up in inverse video (black/white or white/black) for 1 full second to indicate that the computer has taken the prediction into account; then the computer puts the slot where it is about to play into inverse video for 1 full second while it proceeds either to withdraw a token from this slot or to place one in it.

Procedure. Participants were tested individually. The five tokens in their slots were presented in the centre of the screen (Figure 2, initial state 21). Participants were given the following instructions: "The computer is going to withdraw and replace tokens on the board according to a rule. You are going to try and guess where it's going to play next and click at the place where you think it will play." Then, the experimenter demonstrated the procedure for the participant, running through about 12 trials and making as many bad predictions ("You see, I guessed wrong there") as good predictions ("I got it right this time"). After the participant had practised for a dozen trials, the experiment, lasting 20 minutes, began.

For each condition, for each participant, and for each trial, the following data were recorded: the position of the trial in the session, the move the participant predicted, the move the computer made, and the delay between the last computer move and a new prediction, measured in $1 / 60$ th of a second. In order to measure performance, we computed correct predictions but also valid predictions. A correct prediction is strictly a prediction of the move the computer actually makes. A valid prediction is a prediction of a legal move, which means a move that does not violate the rule but is not necessarily the one the computer actually makes. For studying rule discovery, performance was measured in the same way for both conditions. At any given time during the game, only two out of the five slots represent a place where a legal move can be made. In the NG condition, the computer may play either one of these two moves. In the SG condition, the computer can only choose the move that brings it closer to the goal. For instance, in running state 17 $(\mathrm{OOxxO})$,

- When the participant predicts "put a token in slot 3", the prediction is invalid because it does not respect the rule and it is also incorrect because the computer could not possibly play there. 
- When the participant predicts "remove the token in slot 1", which corresponds to moving into state 16 (OOxxx), the prediction is valid because it respects the rule regardless of the move the computer actually makes.

- If the computer does in fact do as the participant predicted and moves into state 16 (OOxxx), then the prediction is correct in addition to being valid.

- However, if the computer moves into state 18 (OOxOO) then the prediction is valid but incorrect.

Performance in both conditions was measured by the number of valid predictions over the total number of trials and, as in P. J. Reber and Kotovsky (1997), by the number of correct predictions over the total number of trials.

\section{Results and discussion}

Results show that participants do learn-correct guesses were above random (Figure 4) - and that rule discovery is in itself as difficult as problem solving. For almost all of the participants, the rule had still not been discovered, even if some learning had occurred, by the end of 180 trials on average.

For each condition, Figure 4 provides the cumulative percentage of valid predictions (top) and the cumulative percentage of correct predictions (bottom) per block of 10 trials. A comparison of performance for all the trials in both experimental groups shows that participants made more valid predictions in the SG condition (.55) than they did in the NG condition $(.33) ; F(1,18)=11.942, p=.002$.

SG participants also showed better performance on correct predictions (.46) than the NG participants $(.23) ; F(1,18)=13.76, p=.001$.

These results suggest that specific goals have a positive effect on rule discovery. We wonder if taking the move's prerequisites into account was the main positive effect of the goal, i.e., participants might be assuming that tokens are placed in slots in order to make it possible to remove the next token. Such predictions have a better chance not only of being compatible with the recursive structure of ChiRP for which the probability of the computer playing a slot doubles from slot 5 to slot $1\left(\mathrm{~S}_{5}=.03 ; \mathrm{S}_{4}=.06\right.$; $\left.\mathrm{S}_{3}=.125 ; \mathrm{S}_{2}=.25 ; \mathrm{S}_{1}=.5\right),{ }^{1}$ but also of being compatible with the goal-tosubgoal structure of the problem (Figure 5).

\footnotetext{
${ }^{1}$ Probability of playing a correct move equals $1 / 32$ when playing token 5 (state 16), equals $2 /$ 32 when playing token 4 (states $23 \& 8$ ), equals $4 / 32$ when playing token 3 , equals $8 / 32$ when playing token 2 , and equals $16 / 32$ when playing token 1 .
} 

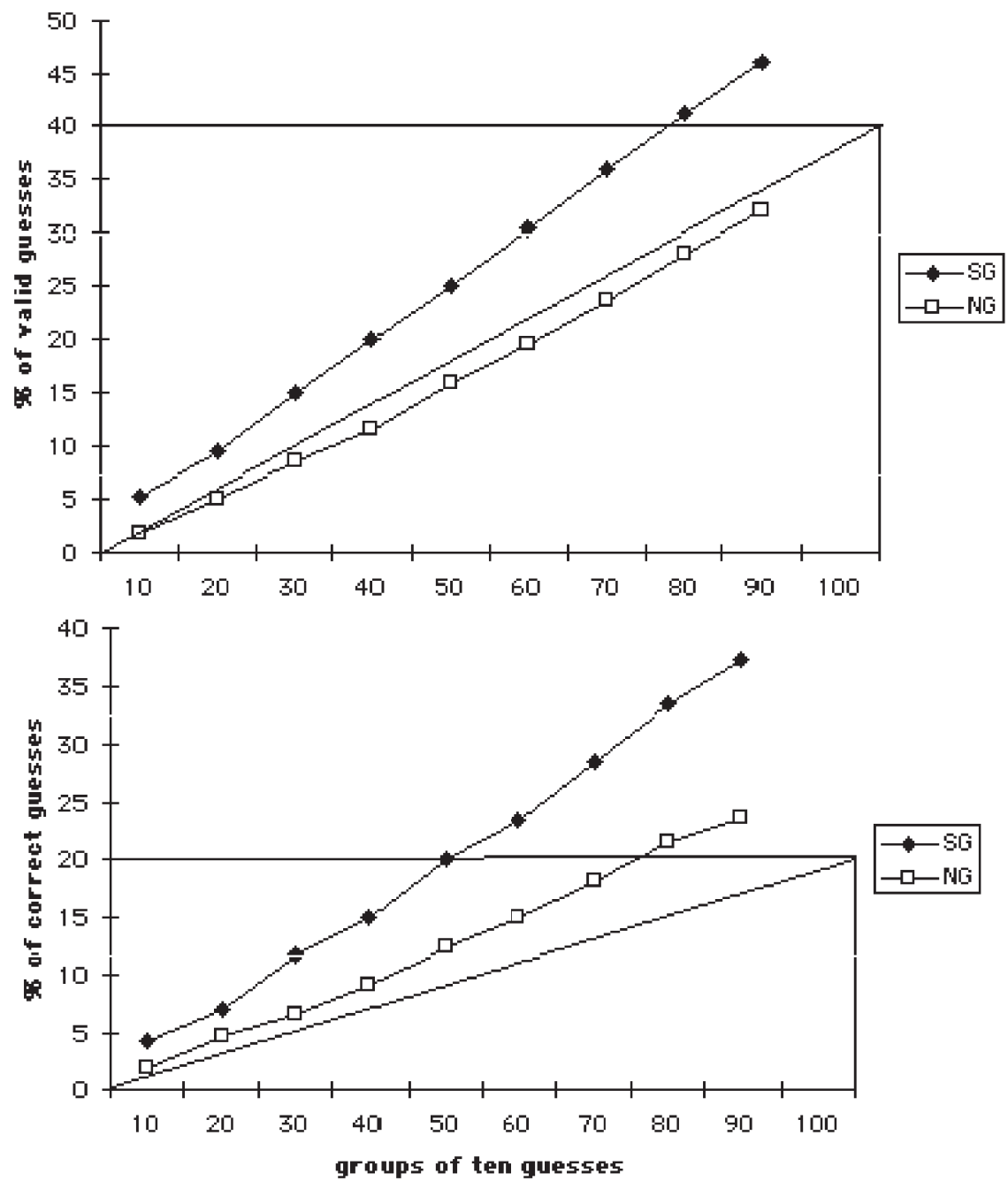

Figure 4. In percentages, the cumulative proportion of valid (top) and correct (bottom) guesses for each successive set of 10 trials for participants when the computer was given a specific goal state (SG) and when the computer was not given a specific goal state (NG). The diagonal corresponds to the probability of random guesses for valid (2 over 5$)$ and correct (1 over 5) moves. 


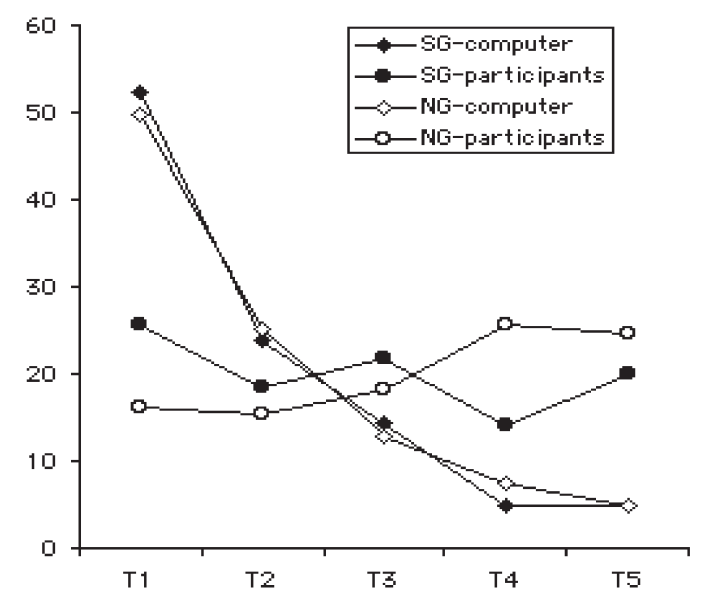

Figure 5. The probability that the computer will play a token in a given slot for specific goal (SG-computer) and nonspecific goal (NG-computer), the observed frequency for participants to choose a token in a given slot when the computer was given a specific goal state (SGparticipants) and when the computer was not given a specific goal state (NG-participants).

Observed frequencies for correct predictions in the NG group (.23) are above random guessing frequencies (.19), but below the frequencies that would have been obtained by guessing in linear progression from slot 5 to 1 (.26).

Indeed, supposing that participants predict a move in any of the five slots at random (.2) the probability of making a valid guess is $.19\left(\mathrm{~S}_{5}=.006\right.$; $\left.\mathrm{S}_{4}=.001 ; \mathrm{S}_{3}=.02 ; \mathrm{S}_{2}=.05 ; \mathrm{S}_{1}=.1\right)$.

Supposing that participants play the five slots in linear progression from slot 5 to slot 1 , then the probability is $.26\left(\mathrm{~S}_{5}=.002 ; \mathrm{S}_{4}=.008 ; \mathrm{S}_{3}=.025\right.$; $\mathrm{S}_{2}=.066 ; \mathrm{S}_{1}=.16$ ).

Supposing that participants play with a good idea of the actual probability $\left(\mathrm{S}_{5}=.03 ; \mathrm{S}_{4}=.06 ; \mathrm{S}_{3}=.125 ; \mathrm{S}_{2}=.25 ; \mathrm{S}_{1}=.5\right)$, the probability of correct predictions would be $.33\left(\mathrm{~S}_{5}=.001 ; \mathrm{S}_{4}=.003 ; \mathrm{S}_{3}=.01 ; \mathrm{S}_{2}=.06\right.$; $\mathrm{S}_{1}=.25$ ).

The SG group obtained a correct prediction frequency (.46) well above the .33 score. The SG group can thus be considered to have acquired, among other things, notions about the goal structure as defined by the rule. For the NG group, results show that the frequencies for choosing one of the five slots were as follows: $\mathrm{S}_{5}=.25 ; \mathrm{S}_{4}=.26 ; \mathrm{S}_{3}=.18 ; \mathrm{S}_{2}=.15 ; \mathrm{S}_{1}=.16$, whereas for the $\mathrm{SG}$ group these frequencies were: $\mathrm{S}_{5}=.20 ; \mathrm{S}_{4}=.14$; $\mathrm{S}_{3}=.21 ; \mathrm{S}_{2}=.18 ; \mathrm{S}_{1}=.26$. The $\mathrm{SG}$ group had a better appreciation of the frequency with which to predict that a move would be made on a given slot (Figure 5). 
TABLE 1

Percentages of moves by the computer for each token in the specific goal and nonspecific goal conditions

\begin{tabular}{lrrrrrr}
\hline & Computer & $T 1$ & $T 2$ & $T 3$ & $T 4$ & $T 5$ \\
\hline Specific goal & & & & & & \\
T1 & 52.38 & 37.63 & 13.21 & 16.70 & 13.00 & 19.45 \\
T2 & 23.81 & 12.33 & 32.09 & 20.23 & 15.81 & 19.54 \\
T3 & 14.29 & 10.47 & 17.05 & 43.80 & 11.24 & 17.44 \\
T4 & 4.76 & 16.28 & 27.91 & 24.42 & 23.26 \\
T5 & 4.76 & 22.09 & 15.12 & 13.95 & 13.95 & 34.88 \\
Nonspecific goal & & & & & & \\
T1 & 49.78 & 20.39 & 12.86 & 15.64 & 25.55 & 25.55 \\
T2 & 24.99 & 10.44 & 23.33 & 17.62 & 24.31 & 24.31 \\
T3 & 12.92 & 11.67 & 12.93 & 28.39 & 25.55 & 21.45 \\
T4 & 7.42 & 18.13 & 10.99 & 20.33 & 28.02 & 22.53 \\
T5 & 4.89 & 12.50 & 12.50 & 15.00 & 30.83 & 29.17 \\
\hline
\end{tabular}

For each token moved by the computer, the corresponding percentages of predictions made by participants for each of the five tokens are shown.

In Table 1, it is clear that NG participants continue to predict slots 4 and 5 more frequently than the other slots. Although these are the slots that most often contain tokens, the computer rarely plays them. They are the ones that SG participants predict the least.

\section{EXPERIMENT 2 : THE EFFECT OF THE GOAL ON MAKING LEGAL MOVES}

The object of the second experiment is to study the effect of the goal on rule discovery in a situation in which participants are asked to move the tokens themselves. We consider valid moves to be a measure of knowing the rule and we measured progressive rule discovery by the increasing percentage of valid moves. In the first Specific Goal, condition (SG), participants are asked to remove all of the tokens from the board. In the second, Nonspecific Goal, condition (NG), participants are only asked to find out how to remove and replace tokens. If the goal effect is one of guiding moves such that participants will try to reduce distance to goal, SG participants should make more errors than NG participants because the goal cannot be reached without making detours (placing tokens in empty slots in order to remove others, something that seems contradictory to the goal of removing them all). SG participants should also have more difficulty in discovering the rule. Overall, SG participants should show poorer performance than NG participants. 


\section{Method}

Participants. A total of 20 participants were recruited from the student body of the University Paris 8-St Denis and assigned in alternating order to one of the two experimental groups: 10 participants were placed in the SG situation and 10 others in the NG situation. None of them had ever seen the problem before.

Materials. A "problem solving" version of ChiRP was developed for Experiment 2, in which tokens can neither be removed nor replaced unless the rule is respected (Figure 2). If the move requested is in contradiction to the rule, the computer does not make the change. The initial state given to the participants is state 21 in which all five slots contain tokens. When the goal is reached, the problem is solved and the experiment is over. If the rule is respected, clicking on a token makes that token disappear and clicking on an empty slot makes a token appear in that slot.

For each trial, the slot that has been chosen by clicking appears on the screen in inverse video for a full second to indicate that the computer has registered the request and then, if the request respects the rule, the computer makes the requested move.

Procedure. Participants were tested individually. The five tokens in their slots were presented in the centre of the screen (Figure 2, initial state 21). SG participants were given the following instructions: "You are going to move tokens on and off the board until you have removed them all'. NG participants were instructed to "Try to move each of the five tokens on and off the board at least once".

The experimenter demonstrated the apparatus by removing the token in slot 1 that can always be removed and then experimentation began. SG experimentation ended either when the participant had reached the goal or when 20 minutes had gone by, whichever came first. NG experimentation ended either when the participant had moved all of the tokens or when 20 minutes had gone by, whichever came first.

For each condition, for each participant, and for each trial, the following data were recorded: the number of the trial, the slot chosen by clicking, whether the move requested consisted of removing or of replacing a token on the board, whether or not the requested move constituted an error, and the time elapsed in $1 / 60$ ths of a second between the previous move and the move in question.

We measured performance by (i) the frequency of moves that respect the rule, a score obtained by dividing the number of valid moves by the total number of moves requested (both legal and illegal), (ii) the 
exploration "Span" (or range), i.e., the number of different states the participant visits at least once, something that corresponds to part of the graph shown in Figure 2, and (iii) the number of trials per "Step", i.e., the mean number of trials it takes a participant to successfully move from one state into another; a score obtained by dividing the number of trials by the exploration span. The frequency of valid moves and the exploration span are implicit learning indicators. The last measure (the number of trials per "Step") is an interesting one because dividing by the span (in which a given state is only counted once regardless of the number of times the participant visits it) allows blocking of the statistical artefact produced by a participant making many legal moves by moving back and forth, in and out of the same states, or inversely, the participant who attains a large exploration span at the cost of many errors. A cautious participant can, for example, obtain a high score for the frequency of legal moves and have a narrow exploration span by making the same successful moves over and over again. Another more daring participant might show a wide exploration span and a low frequency of legal moves because he or she accepts making many errors in the course of attempting to move a given token.

Finally, we would like to specify that a single error repeated consecutively - when, for example, at state "OOOOO", the participant clicks twice on slot 5-is counted as a single error.

\section{Results and discussion}

ChiRP is a difficult problem for participants who are asked to solve it. After 20 minutes, only 3 participants out of the 10 in the SG group were able to solve the problem, and this in 228, 282, and 764 moves respectively. The other SG participants were not able to solve the problem although the mean number of trials for these 7 participants was 274 (legal and erroneous moves). The 10 participants in the NG group performed a mean of 320 trials and 4 of them reached the goal state although they were not asked to do so.

Although there was no significant difference between the SG and the NG participants for the time required to take a Step in the span, i.e., to visit a new state, the results shown in Figure 6 indicate that the frequency of making legal moves is higher for $\mathrm{NG}$ participants $(\mathrm{NG}=.575)$ than for $\mathrm{SG}$ participants $(\mathrm{SG}=.474) ; F(1,18)=4.89, p=.04$.

Although knowledge of the goal seems to help participants discover the rule in Experiment 1 when they are asked to predict the moves of a computer that is playing towards the goal, it is a hindrance when participants are asked to play and reach this goal themselves. Our 

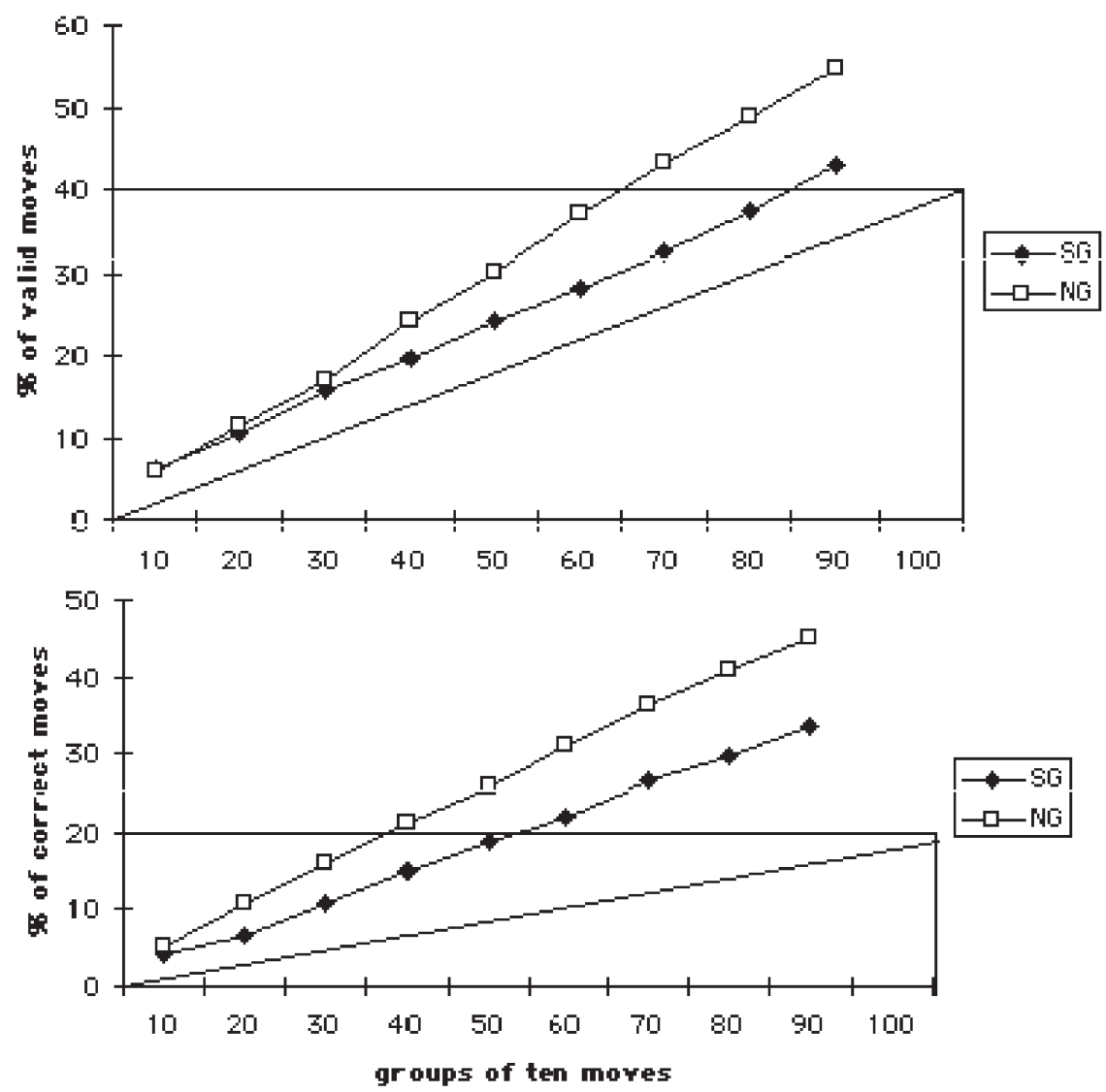

Figure 6. In percentages, the cumulative proportion of valid (top) and correct (bottom) moves for each successive set of 10 trials for participants who were given a specific goal state (SG) and participants who were given a nonspecific goal state $(\mathrm{NG})$. The diagonal corresponds to the probability of random guesses for legal ( 2 over 5$)$ and correct (1 over 5) moves.

hypothesis is that reducing the distance to goal mobilises the attention of SG participants and encourages them to reject the correct moves that they perceive as moves which increase distance to goal. If this is the case, the number of errors SG participants commit in attempting to remove a token should be greater than the number they commit in attempting to replace one. Because in attempting to reduce distance to goal, participants will focus their efforts on removing tokens, while the rule, counterintuitive as it is, requires replacing tokens in order to remove others. The results are consistent with this hypothesis: in the SG condition, the probability of making moves that do not respect the rule is 
higher for removing tokens (.62) than it is for replacing them (.38); $F(1$, $62)=5.3, p=.02$ ). No such significant difference can be observed for the NG group: the rate of errors linked to attempts to remove tokens is .47 versus .53 for replacing them; $F(1,62)=0.52$, NS.

\section{GENERAL DISCUSSION}

In order to study the goal effect on problem solving, we compared the behaviours of participants playing ChiRP for 20 minutes through move analysis.

In Experiment 1, we devised a situation close to situations of implicit learning. A typical experiment in implicit learning is the one carried out by Perruchet and Amorim (1992): participants are asked to guess in which one of four locations (A, B, C, D) a light will appear, without knowing that there is an underlying pattern: DBCACBDCBA. One can picture the sequence that goes from the initial state of ChiRP to the goal state (Figure 2) as one such pattern, if the computer is making moves and if the participant's task is to guess which token the computer will move.

In Experiment 2, the participant is the one who makes the moves. In Experiment 1, when the participant makes an illegal prediction, the computer nevertheless makes a legal move and the state changes, whereas in Experiment 2, when the participant tries to make an illegal move, the move is not made and the state remains the same. Thus, in Experiment 2, illegal move probabilities decrease after having made an error because the state does not change and the participant can choose a different move. Nevertheless, participants make more correct predictions of computer's legal moves in Experiment 1 than legal moves in Experiment 2. For instance, when the goal is given in Experiment 1, 55\% of the trials are predictions of legal moves, versus $47 \%$ of legal moves in Experiment 2. We hypothesised that the goal effect is different in these two experiments.

In order to attribute a meaning to something someone does, it is necessary to know (or to imagine) what his or her specific goal is. For instance, in $\mathrm{TOH}$, if one knows that the goal is to put the largest disk on peg $\mathrm{C}$, and if an expert in the game begins by putting the smallest disk on $\mathrm{C}$, one might conclude that this move is done in order to reach the goal. If the expert then puts the medium-sized disk on B, and then the smaller disk on top of that one on $\mathrm{B}$, one might conclude that she has done so in order to free the largest disk and is about to move it to peg C. The order in which the expert has placed the medium-sized and the smallest disk on B might suggest that she is following a rule, or a pattern, of some sort: "only one disk can be moved at a time", "only the top disk on a peg can be moved" and "a larger disk cannot be placed on a smaller disk". 
Knowing that there is in fact a specific goal to reach should make rule discovery easier, and this is the case in Experiment 1. In the condition in which the computer plays towards the "xxxxx" goal and the participants know that it is doing so, performance is improved: $55 \%$ of the predictions are valid versus $33 \%$ valid when the computer makes legal moves either towards or away from the xxxxx state.

In Experiment 1, the participants need take no action, they do not have to make the moves themselves. And when there is a specific goal, the participants do not have to try to reach it-the computer is the one that works at reaching it. In Experiment 2, participants must take action themselves by moving the tokens, and when there is a specific goal they have to reach it themselves even though they do not know what the rule for moving the tokens is. We hypothesised that knowing the specific goal should inhibit rule discovery.

Indeed, solving any problem requires reducing the distance to goal. For instance, in TOH, the goal "Move the tower from A to C" could be reached in one move by picking up the stack of disks and moving them all to peg $\mathrm{C}$. But as in all puzzle-like problems, instant distance to goal reduction is not possible. The rules given in the instructions provide constraints that oblige problem solvers to make detours. When they do not know or do not understand the rules, they try reducing distance to goal in the most straightforward fashion, and when this fails, they try reducing the distance in some other way, without profiting from what might be learned by this failure because their attention is focused elsewhere, on the specific goal to reach. Thus, they might be not paying attention to subgoals' prerequisites.

However, when participants have no specific goal but do have the task of trying to move each and every one of the tokens, they learn the prerequisites for moving tokens. These prerequisites are what the rules express.

This is what we observed for Experiment 2. In the condition in which participants are given a specific goal, performance deteriorated: $47 \%$ of the moves corresponded to legal moves versus $57 \%$ when participants were given a nonspecific goal.

These results are consistent with our approach to puzzle-like problem solving. In the constraints model developed by Richard (Richard et al., 1993), we argue that most of the difficulties in problem solving do in fact spring from misunderstanding the rules as expressed in the instructions and from the way in which the participant constructs the goal.

The constraint model describes misunderstanding using a list of constraints in the form of "DO NOT (Action) ON (Object)". This list of constraints filters possible moves and allows prediction of the moves a participant is going to perform. Feedback and, above all, states for which the filter blocks all moves (impasses), delete the implicit constraints. In this way, throughout the problem-solving process, the participant continually 
adjusts his or her list of constraints and this entails adjusting his or her representation of the problem. By doing so, we explain the exploratory phase that is found in the protocols (Figure 3). We did not provide the participant with the rules to the problem in order to study rule discovery.

In the constraints model, decision making is goal-oriented: moves are constrained by the goal. The model predicts that the representation of the goal can affect rule discovery because the rules indicate the legality of moves and, by restricting moves, the way to construct the goal.

These results are also consistent with the results that show that having a specific goal impairs learning (Geddes \& Stevenson, 1997; Sweller, 1998; Vollmeyer et al., 1996). However, Experiment 1 shows that a specific goal can improve performance when participants are not acting by themselves, but having to predict moves.

Experiment 1 is one in which participants have no specific goal to reach: participants are asked to predict the computer's next move and the computer either moves through the problem space directly towards the specific goal or it moves back and forth through the problem space without attempting to reach the specific goal. When the computer is going for the specific goal, participants can interpret its moves as necessary moves to reach it and can thus eliminate their own erroneous interpretations. For example, a participant might begin by thinking that the computer is going to withdraw each of the tokens one by one. This conception will be eliminated when the participant sees that the computer adds tokens in order to withdraw others. When the computer plays aimlessly, participants are not in a position to interpret why the computer adds or withdraws tokens. In other words, the SG condition in Experiment 1 provides participants with the opportunity to attribute an intention to the actions they observe.

Experiment 2 is one in which participants act by themselves. In this case, there is a negative goal effect because the initial representation of the specific goal that participants try to construct step by step is mistaken. With the mistaken goal representation in sight, participants focus on removing tokens rather than on how to remove them. This is the likely explanation for the difference in distribution of errors for withdrawing versus replacing tokens. Not only do participants make more errors in the SG condition, but these errors show a lopsided distribution: $68 \%$ of them are made in attempting to remove a token versus only $47 \%$ made in attempting to add a token. A similar lopsided distribution was found for children: with the goal, $71 \%$ of the errors are "withdrawal" errors and 57\% are "adding" errors (Zanga \& Tijus, 2001).

The results of this research also have implications for implicit learning. In Experiment 1, participants showed progressively increasing accuracy of prediction even though they were not able to verbalise the rule. This would seem to indicate that a distinction should be made between implicit 
learning situations in which a specific goal is provided and situations where one is not. It also indicates that a further distinction should be made between situations in which participants are required to take action themselves and situations in which they are only asked to observe and predict. One consequence of making these distinctions is that knowing or not knowing that there is a specific goal influences "intentional" versus "incidental" situations of implicit learning. This prediction is compatible with Berry and Broadbent (1988): when they provided participants with specific goals, performance deteriorated when participants were also told there was a rule. It is possible that this effect is due to focus on the specific goal.

\section{CONCLUSION}

Solving a problem entails learning how to apply a set of rules towards reaching a goal. Despite the discussion in Shanks and St. John (1994) that problem solving and implicit learning both present situations in which participants learn to apply one or more rules that they usually cannot verbalise, rule discovery in problem solving has rarely been studied.

What we have tried to bring out in this article is the different roles the problem goal plays in rule discovery: it slows rule discovery when participants are so focused on it that they neglect the rule, which is to say, what makes it possible to make moves. But the goal also furnishes an opportunity to discover the rules because it gives a meaning to the actions one does in order to reach it. If I am trying to reach xxxxx and I am in xxxOx, I might try removing token $\mathrm{t} 2$ and thus make an error. But if I am in $\mathrm{xxxOx}$ and $\mathrm{I}$ can see that token $\mathrm{t} 1$ has to be added, $\mathrm{xxxOO}$, in order to remove token $\mathrm{t} 2, \mathrm{xxxxO}$, and then token $\mathrm{t} 1, \mathrm{xxxxx}$, the meaning of "adding t1" is justified by "removing t2". The goal we are talking about is the specific problem goal, which is to say a goal state to be reached. It is to know for a fact that participants always have a goal, that we might name a subject goal, and that our participants in the so-called nonspecific goal group (NG) do have the goal of "Trying to move each of the five tokens at least once". Note that the rule can be found when participants are presented states in isolation and the token that can be moved on and off (Zanga, 1997, Zanga \& Tijus, 2001; Zanga, Tijus, \& Richard, 2001).

Although it will be informative to observe SG participants having NG stimuli, and the reverse, our study provides two ideas. The first idea is that a good heuristic for problem solving seems to be to explore in order to discover the rules that determine what can and cannot be done before trying to reach a specific goal. The second idea is that understanding a problem entails (i) attention and implicit learning for taking feedback into account, and (ii) interpreting feedback in the light of the goal. 
In conclusion, what this study in problem solving can bring to implicit learning is that rules cannot be applied directly to the material. To solve a problem, one has to interpret general rules and apply them to specific instances (Richard \& Verstiggel, 1990). For instance in the Missionaries and Cannibals problem (M\&C), where three missionaries and three cannibals initially on the left bank of a river must be transported to the right bank, the rule is "No missionaries may ever be left with a larger number of cannibals on either bank or in the boat". Is the problem state with three missionaries on the left side and three cannibals on the right side of the river a state that respects the rule? Aren't there more cannibals than missionaries on the right bank? This is a legal state because it must be understood that the rule about there never being "more" cannibals only applies when there is at least one missionary. Knowing that cannibals eat missionaries helps in selecting the proper interpretation of the rule. However, if the cannibals are replaced by white tokens and the missionaries by black tokens, the correct interpretation of the rule is made more difficult (Richard et al., 2002).

Manuscript received June 2002

Revised manuscript received June 2003

\section{REFERENCES}

Anzaï, Y., \& Simon, H. A. (1979). The theory of learning by doing. Psychological Review, 86, $124-140$.

Berry, D. C., \& Broadbent, D. E. (1984). On the relationship between task performance and associated verbalizable knowledge. Quarterly Journal of Experimental Psychology, 36, 209231.

Berry, D. C., \& Broadbent, D. E. (1988). Interactive tasks and the implicit-explicit distinction. British Journal of Psychology, 79, 251-272.

Clément, E., \& Richard, J. F. (1997). Knowledge of domain effects in problem representation: The case of Tower of Hanoi isomorphs. Thinking and Reasoning, 3, 133-157.

Duncker, K. (1945). On problem solving. Psychological Monographs, 58, (Whole No. 270).

Geddes, B. W., \& Stevenson, R. J. (1997). Explicit learning on a dynamic system with a nonsalient pattern. Quarterly Journal of Experimental Psychology, 50A (4), 742-765.

Jeffries, R., Polson, P. G., \& Razran, R. (1977). A process model for missionaries-cannibals and other river-crossing problems. Cognitive Psychology, 17, 248-294.

Kohler, W. (1964). Psychologie de la Forme. Paris: Editions Gallimard.

Kotovsky, K., Hayes J. R., \& Simon, H. A (1985). Why are some problems hard? Evidence from Tower of Hanoi. Cognitive Psychology, 17, 248-294.

Kotovsky, K., \& Kushmerick, N. (1991). Processing constraints and problem difficulty: A model. In K. J. Hammond \& D. Gentner (Eds.), Proceedings of the 13th Annual Conference of the Cognitive Science Society, August, Chicago. Hillsdale, NJ: Lawrence Erlbaum Associates, Inc.

Kotovsky, K., \& Simon, H. A. (1990). What makes some problems really hard: Explorations in the problem space of difficulty, Cognitive Psychology, 22, 143-183.

Lewicki, P., Hill, T., \& Czyzewska, M. (1992). Nonconscious acquisition of information. American Psychologist, 47, 796-801. 
Lockhart, R. S., Lamon, M., \& Gick, M. L. (1988). Conceptual transfer in simple insight problems. Memory \& Cognition, 16, 36-44.

MacGregor, J. N., Ormerod, T. C., \& Chronicle, E. P. (2001). Information processing and insight: A process model of performance on the nine-dot and related problems. Journal of Experimental Psychology: Learning, Memory and Cognition, 27, 176-201.

Mathews, R. C., Buss, R. R., Chinn, R., \& Stanley, W. B. (1988). The role of explicit and implicit learning processes in concept discovery. Quarterly Journal of Experimental Psychology, 40, 135-165.

Mayer, R. E. (1983) Thinking, problem solving, cognition. New York: Freeman \& Co.

Newell, A., \& Simon, H. A. (1972). Human problem solving. Englewood Cliffs, NJ: Prentice Hall.

O'Hara, K. P., \& Payne S. J. (1998). The effects of operator implementation cost on planfulness of problem solving and learning. Cognitive Psychology, 35, 34-70.

Ohlsson, S. (1984a). Restructuring revisited, I. Summary and critique of the Gestalt theory of problem solving. Scandinavian Journal of Psychology, 25, 65-78.

Ohlsson, S. (1984b). Restructuring revisited, II. An information processing theory of restructuring and insight. Scandinavian Journal of Psychology, 25, 117-129.

Perruchet, P., \& Amorim, M. A. (1992). Conscious knowledge and changes in performance in sequence learning: Evidence against dissociation. Journal of Experimental Psychology, 18, $785-800$.

Perruchet, P., Gallego, J., \& Pacteau, C. (1992). A reinterpretation of some earlier evidence for abstractiveness of implicitly acquired knowledge. Quarterly Journal of Experimental Psychology, 44, 193-210.

Reber, A. S. (1989). Implicit learning and tacit knowledge. Journal of Experimental Psychology: General, 118, 219-235.

Reber, A. S., Kassin, S. M., Lewis, S., \& Cantor, G. (1980). On the relationship between implicit and explicit modes in the learning of a complex rule structure. Journal of Experimental Psychology: Human and Learning Memory, 6, 492-502.

Reber, P. J., \& Kotovsky, K. (1997). Implicit learning in problem solving: The role of working memory capacity. Journal of Experimental Psychology: General, 126, 178-203.

Richard, J. F. (1982). Planification et organisation des actions dans la résolution du problème de la tour de Hanoi par des enfants de 7 ans. L'Année Psychologique, 82, 307-336.

Richard, J. F., Clément, E., \& Tijus, C. A. (2002). Les différences de difficulté dans la résolution des problèmes isomorphes comme révélatrices des composantes sémantiques dans la construction de la représentation du problème. In J. Pitrat (Ed.), Hommage à H. Simon, Revue d'Intelligence Artificielle, 16, 1-2, 191-219.

Richard, J. F., Poitrenaud S., \& Tijus, C. (1993). Problem-solving restructuration: Elimination of implicit constraints. Cognitive Science, 4, 497-529.

Richard, J. F., \& Verstiggel, J. C. (1990). La représentation de l'action dans les processus de compréhension. Langage, 100, 115-126.

Shanks, D. R., \& St. John, M. F. (1994). Characteristics of dissociable human learning systems. Behavioral and Brain Sciences, 17(3), 367-447.

Simon, H. A. (1979). Models of thought. New Haven, CT: Yale University Press.

Simon, H. A., \& Lea, G. (1974). Problem solving and rule induction: A unified view. In L. W. Gregg (Ed.), Knowledge and cognition (pp. 105-128). Potomac, MD: Lawrence Erlbaum Associates Inc.

Sweller, J. (1988). Cognitive load during problem solving: Effects on learning. Cognitive Science, $12,257-285$.

Sweller, J., Mawer, R. F., \& Ward, M. R. (1983). Development of expertise in mathematical problem solving. Journal of Experimental Psychology: General, 4, 639-661. 
Tijus, C. A. \& Najab, F. (1986). Pour en finir avec le problème des 9 points, le problème des onze points. Communication affichée au Colloque de la Société Française de Psychologie sur "Les apprentissages: perspectives actuelles". Université de Paris 8, France.

Tijus, C. A., \& Santolini, A. (1996). How to change mental constructs. Journal of Psychology, $130(4), 401-413$.

Vollmeyer, R., Burns, B. D., \& Holyoak, K. J. (1996). The impact of goal specificity on strategy use and the acquisition of problem structure. Cognitive Science, 20, 75-100.

Weisberg, R. W., \& Alba, J. W. (1981). An examination of the alleged role of fixation in the solution of several insight problems. Journal of Experimental Psychology: General, 110(2), $169-192$.

Zanga, A. (1997). L'apprentissage implicite en résolution de problème. Thèse de doctorat, Paris VIII, France.

Zanga, A., \& Tijus, C. (2001). Dissociation entre apprentissage et verbalisation en situation de résolution de problèmes. Archives de Psychologie, 68, 233-258.

Zanga, A., Tijus, C. A., \& Richard, J. F. (2001). Apprentissage implicite en situation de découverte de règle et de résolution de problème. Bulletin de Psychologie, 4, 389-399. 